\title{
Assessment of left atrial volume before and after pulmonary thromboendarterectomy in chronic thromboembolic pulmonary hypertension
}

\author{
Nicholas A Marston', William R Auger ${ }^{1}$, Michael M Madani', Bruce J Kimura², G Monet Strachan', \\ Ajit B Raisinghani ${ }^{1}$, Anthony N DeMaria ${ }^{1}$ and Daniel G Blanchard ${ }^{1 *}$
}

\begin{abstract}
Background: Impaired left ventricular diastolic filling is common in chronic thromboembolic pulmonary hypertension (CTEPH), and recent studies support left ventricular underfilling as a cause. To investigate this further, we assessed left atrial volume index (LAVI) in patients with CTEPH before and after pulmonary thromboendarterectomy (PTE).

Methods: Forty-eight consecutive CTEPH patients had pre- \& post-PTE echocardiograms and right heart catheterizations. Parameters included mean pulmonary artery pressure (mPAP), pulmonary vascular resistance (PVR), cardiac index, LAVI, \& mitral E/A ratio. Echocardiograms were performed $6 \pm 3$ days pre-PTE and $10 \pm 4$ days post-PTE. Regression analyses compared pre- and post-PTE LAVI with other parameters.

Results: Pre-op LAVI (mean $19.0 \pm 7 \mathrm{~mL} / \mathrm{m}^{2}$ ) correlated significantly with pre-op PVR $(R=-0.45, p=0.001$ ), $\mathrm{mPAP}$ $(R=-0.28, p=0.05)$ and cardiac index $(R=0.38, p=0.006)$. Post-PTE, LAVI increased by $18 \%$ to $22.4 \pm 7 \mathrm{~mL} / \mathrm{m}^{2}$ $(p=0.003$ ). This change correlated with change in PVR (765 to 311 dyne-s/cm,$p=0.01$ ), cardiac index (2.6 to $\left.3.2 \mathrm{~L} / \mathrm{min} / \mathrm{m}^{2}, p=0.02\right)$, and $\mathrm{E} / \mathrm{A}(.95$ to $1.44, p=0.002)$.

Conclusion: In CTEPH, smaller LAVI is associated with lower cardiac output, higher mPAP, and higher PVR. LAVI increases by $\sim 20 \%$ after PTE, and this change correlates with changes in PVR and mitral E/A. The rapid increase in LAVI supports the concept that left ventricular diastolic impairment and low E/A pre-PTE are due to left heart underfilling rather than inherent left ventricular diastolic dysfunction.
\end{abstract}

Keywords: Left atrial volume, Chronic thromboembolic pulmonary hypertension, Pulmonary thromboendarterectomy

\section{Background}

Chronic thromboembolic pulmonary hypertension $(\mathrm{CTEPH})$, the result of unresolved thrombus in the pulmonary vasculature following pulmonary embolism, carries a high rate of morbidity and mortality if left untreated [1-3]. In addition to the well-described right-sided dysfunction seen in CTEPH, left ventricular (LV) diastolic impairment is also common. Diminished early diastolic filling of the LV is frequently present, resulting in reversal

\footnotetext{
* Correspondence: dblanchard@ucsd.edu

'School of Medicine and Sulpizio Cardiovascular Center, University of California San Diego, 9444 Medical Center Drive, \#7411, La Jolla, CA 92037, USA

Full list of author information is available at the end of the article
}

of the "E" (early rapid filling) and "A" (atrial kick) velocities in mitral inflow.

Several theories have been put forth to explain this LV diastolic "dysfunction", including decreased LV volume and compliance due to leftward septal shifting, and true intrinsic dysfunction of the LV [4]. More recent studies, however, have supported the theory of decreased RV output and relative LV underfilling as the primary cause of the perceived LV diastolic impairment in CTEPH [5-7]. If this is correct, one might hypothesize that left atrial volume would increase after successful pulmonary thromboendarterectomy (PTE) and potentially correlate with improvements in right heart catheterization measurements. We investigated this in a relatively large 
group of patients with CTEPH referred for PTE at our institution.

\section{Methods}

\section{Patient population}

This is a retrospective analysis of forty-eight consecutive CTEPH patients undergoing PTE at a large CTEPH referral center. The study cohort was comprised of 24 men and 24 women with a mean age of $52 \pm 16$ years, ranging from 19 to 84 . All patients had class III to IV symptoms according to the New York Heart Association (NYHA) Functional Classification. Pre- and postoperative echocardiograms and right heart catheterizations were performed in each case. All PTE surgeries were completed at UCSD Medical Center using methods outlined in previous publications [8]. The UCSD institutional research review committee approved the study.

\section{Echocardiography}

Echocardiograms were performed $6 \pm 3$ days before and $10 \pm 4$ days following PTE. A Vivid cardiovascular ultrasound system (GE VingMed, Horton, Norway) was used for all cases. Studies included measurements of the left atrial volume (LAVI), mitral E/A ratio, and mitral annular $\mathrm{E} / \mathrm{E}$ ' ratio. Measurements of the left ventricle were also acquired, including cardiac index $(\mathrm{CI})$ and end-systolic and end-diastolic diameters. All echocardiographic techniques followed the recommendations of the American Society of Echocardiography [9]. Maximum left atrial volume was measured at end-systole and then indexed using body surface area, a technique that has demonstrated the strongest correlation to cardiovascular risk stratification [10].

\section{Right heart catheterization}

Right heart catheterization (RHC) using a Swan-Ganz catheter was performed within 48 hours of the preoperative echocardiogram ( $6 \pm 5$ days prior to surgery) and on post-operative day 1 . Invasive measurements included mean pulmonary artery pressures (mPAP), pulmonary vascular resistance (PVR), pulmonary capillary wedge pressure (PCWP), and cardiac output. All pressure measurements were obtained at end-expiration; three measurements were recorded for each variable and then averaged for a final value. Cardiac output was calculated using thermodilution; again, three measurements were obtained and averaged for a final value. PVR was calculated using the formula: PVR $=80$ (mPAP-mPCWP) $/$ cardiac output.

\section{Statistical analysis}

Pre- and post-operative values are expressed as mean \pm standard deviation. Differences in left atrial volume and other characteristics before and after PTE were evaluated using a two-tailed paired Student's t-test. A p value $<0.05$ was considered statistically significant. Linear regression analyses were performed to determine relationships between LA volume and other cardiac parameters using online regression software (http://www. wessa.net) [11].

\section{Results}

Prior to PTE all patients had evidence of significantly elevated right-sided pressures [mPAP of $45.5 \pm 9.6 \mathrm{mmHg}$ and PVR of $765 \pm 396$ (dyne-sec) $\left./ \mathrm{cm}^{5}\right]$, and many had depressed cardiac output (mean CO of $3.9 \pm 1.2 \mathrm{l} / \mathrm{min}$ ). Evidence of diastolic dysfunction was also present, with decreased mitral E/A ratio $(0.95 \pm 0.31)$ and increased deceleration time $(224 \pm 60 \mathrm{~ms})$. Mitral lateral annular $\mathrm{E} / \mathrm{E}^{\prime}$ was $6.1 \pm 2.8$. Of note, pre-operative left atrial volume index was relatively low $\left(19.0 \pm 7.0 \mathrm{~cm}^{3} / \mathrm{m}^{2}\right)$. All baseline hemodynamic and echocardiographic findings are listed in Table 1.

Following surgery significant improvements in hemodynamics were observed. The mean pulmonary arterial pressure fell to $28.8 \pm 6.7 \mathrm{mmHg}(\mathrm{p}<0.001)$ and pulmonary vascular resistance dropped to $310 \pm 143$ (dyne-sec) $/ \mathrm{cm}^{5}$ $(\mathrm{p}<0.001)$. This normalization of right-sided pressures coincided with an improvement in cardiac output to $5.2 \pm$ $1.6 \mathrm{~L} / \mathrm{min}(\mathrm{p}<0.001)$. Further, LV diastolic function improved post-operatively, with the E/A ratio rising to $1.45 \pm 0.48(\mathrm{p}<0.001)$ and the $\mathrm{E}$ deceleration time dropping into the normal range $(189 \pm 39 \mathrm{~ms})(\mathrm{p}<0.001)$. A significant increase in left atrial volume index also occurred (from $19.0 \pm 7.0$ to $\left.22.4 \pm 6.8 \mathrm{~cm}^{3} / \mathrm{m}^{2}\right)(\mathrm{p}=0.003)$. Mitral annular E/E' increased to $7.9 \pm 2.7(\mathrm{p}<0.001)$. The changes in each variable following surgery are listed in Table 1.

Left atrial volume not only increased with surgery but also correlated well with established CTEPH parameters. In the pre-operative state, LA volume index was inversely correlated with PVR $(R=-0.45, p=0.001$, Figure 1$)$, and $\operatorname{mPAP}(\mathrm{R}=-0.28, \mathrm{p}=0.05)$, and was positively correlated

Table 1 Hemodynamic and echocardiographic characteristics pre- and post-PTE

\begin{tabular}{lccc}
\hline & Pre-PTE & Post-PTE & p value \\
\hline Right heart catheterization & & & \\
Mean PA pressure $(\mathrm{mmHg})$ & $45.5 \pm 9.6$ & $28.8 \pm 6.7$ & $<0.001$ \\
PVR (dyne-sec)/cm ${ }^{5}$ & $765 \pm 396$ & $310 \pm 143$ & $<0.001$ \\
Cardiac output $(\mathrm{L} / \mathrm{min})$ & $3.9 \pm 1.2$ & $5.2 \pm 1.6$ & $<0.001$ \\
Echocardiogram & & & \\
Cardiac index & $2.6 \pm 0.8$ & $3.2 \pm 0.5$ & $<0.001$ \\
Mitral E/A ratio & $0.95 \pm 0.31$ & $1.45 \pm 0.48$ & $<0.001$ \\
Lateral E/E' ratio & $6.1 \pm 2.8$ & $7.9 \pm 2.7$ & $<0.001$ \\
Deceleration time $(\mathrm{ms})$ & $224 \pm 60$ & $189 \pm 39$ & $<0.001$ \\
Left atrial volume index $\left(\mathrm{cm}^{3} / \mathrm{m}^{2}\right)$ & $19.0 \pm 7.0$ & $22.4 \pm 6.8$ & 0.003 \\
\hline
\end{tabular}




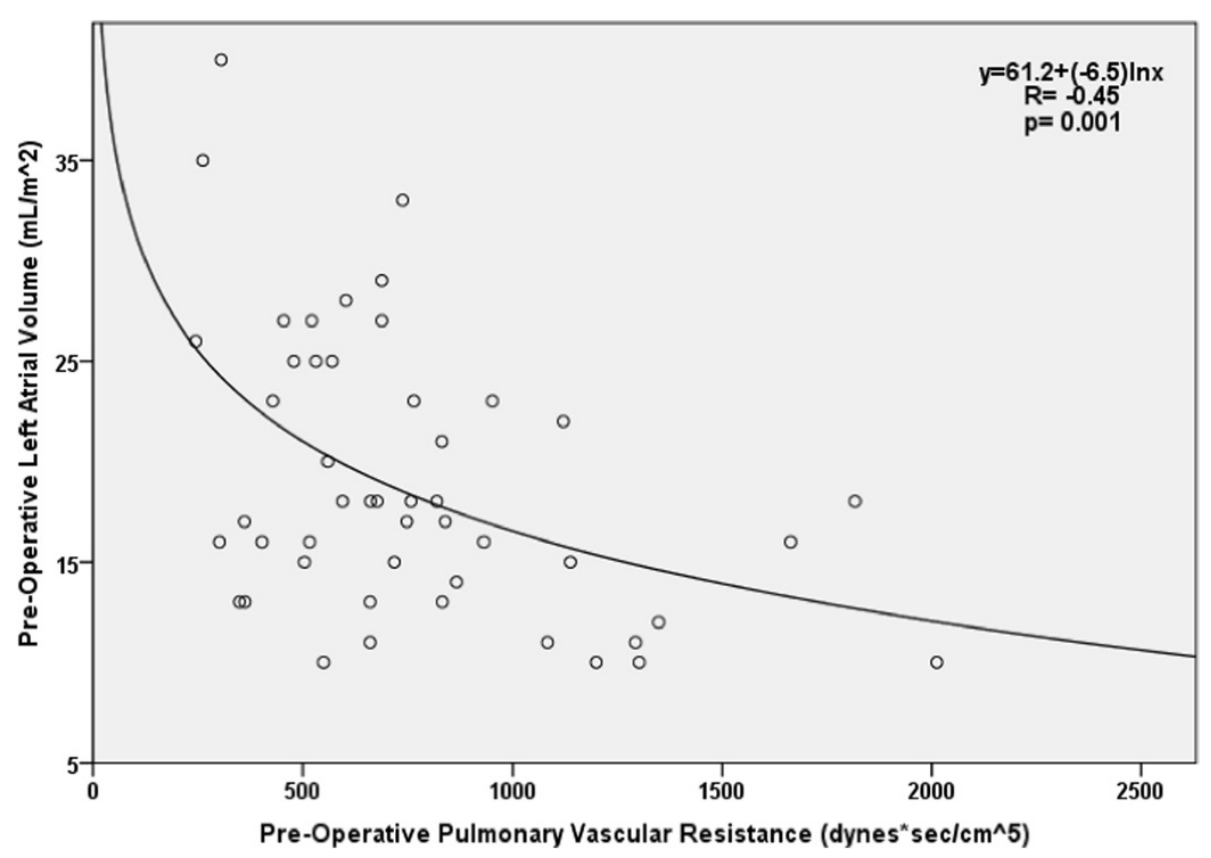

Figure 1 Regression analysis of pre-operative LA volume index vs. PVR.

with cardiac index $(\mathrm{R}=0.48, \mathrm{p}=0.001)$. Smaller pre-op LA volumes also correlated with markers of diastolic dysfunction, such as greater $E$ deceleration times $(R=-0.34$, $\mathrm{p}=0.009)$ and lower mitral annular $\mathrm{E} / \mathrm{E}^{\prime}(\mathrm{R}=0.36$, $\mathrm{p}=0.03)$.

Post-PTE, the change seen in LA volume index was associated with improvement in many of these same parameters. LA volume change correlated significantly with decrease in PVR $(\mathrm{R}=-0.36, \mathrm{p}=0.01)$ and increase in cardiac index $(R=0.41, p=0.007)$. Similarly, increasing LA volume index correlated with improving diastolic function, such as an increase in mitral $\mathrm{E} / \mathrm{A}$ ratio $(\mathrm{R}=$ $0.44, \mathrm{p}<0.001$, Figure 2$)$ and $E / E^{\prime}(R=0.36, p=0.03)$. All correlation coefficients are listed in Table 2.

\section{Discussion}

The relevance of left atrial volume and function in overall cardiovascular health has been increasingly recognized in the last several years. LA volume is now understood to be a noninvasive "biomarker" that can predict cardiovascular risk and prognosis in a wide range of cardiovascular diseases [12]. At a physiologic level, the contribution of

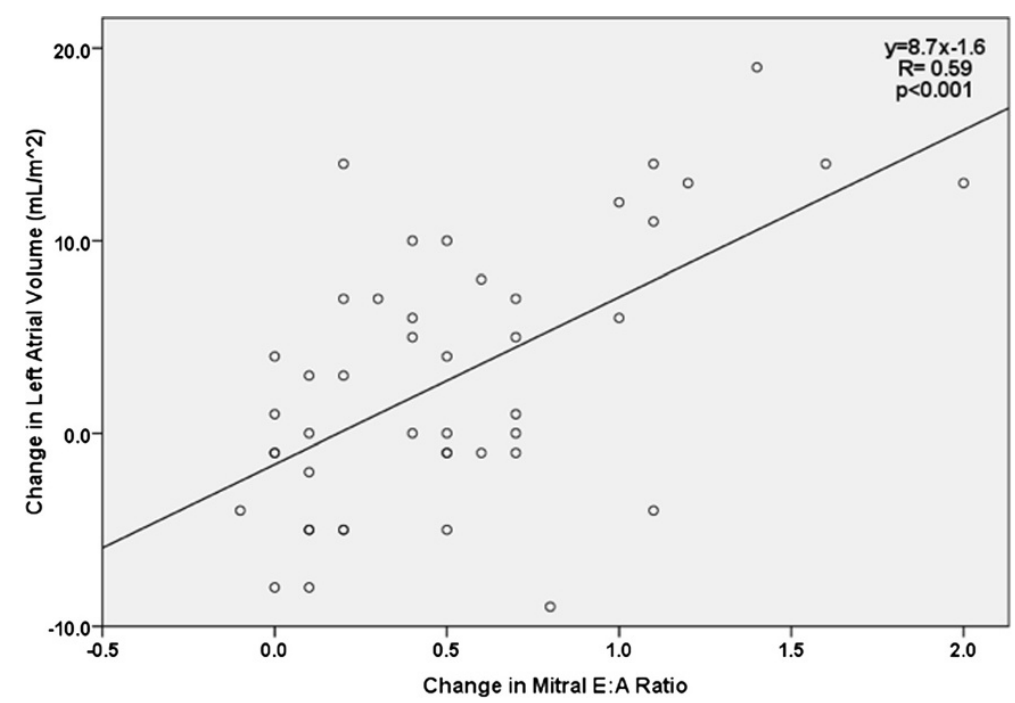

Figure 2 Regression analysis of change in LA volume vs. change in mitral E/A ratio. 
Table 2 Correlation coefficients and $p$ values for left atrial volume vs. RHC and echo parameters

\begin{tabular}{lcc}
\hline & R & p value \\
\hline Pre-PTE & -0.45 & \\
LAVI vs PVR & -0.28 & 0.001 \\
LAVI vs mPAP & 0.39 & 0.05 \\
LAVI vs CO & 0.48 & 0.006 \\
LAVI vs CI & 0.13 & 0.001 \\
LAVI vs Mitral E/A ratio & 0.36 & 0.41 \\
LAVI vs E/E' ratio & -0.34 & 0.03 \\
LAVI vs Decel time & & 0.009 \\
Change after PTE & -0.36 & \\
LAVI vs PVR & -0.06 & 0.01 \\
LAVI vs mPAP & 0.08 & 0.68 \\
LAVI vs CO & 0.41 & 0.93 \\
LAVI vs CI & 0.44 & 0.007 \\
LAVI vs Mitral E/A ratio & 0.36 & $<0.001$ \\
LAVI vs E/E' ratio & -0.24 & 0.03 \\
LAVI vs Decel time & & 0.11 \\
\hline
\end{tabular}

the left atrium to the performance of the left ventricle is well established [13], but alterations in this relationship for different disease states remain an area of interest.

This study is the first to examine left atrial volume in patients with CTEPH before and after PTE. We demonstrated that LA volumes are in the low-normal range in this population, and that LAVI significantly increases following PTE. Furthermore, smaller LA volumes before surgery are a marker for disease severity, and are associated with higher PVR, higher mPAP, and lower CO. This suggests that LA volume may have a role in the evaluation of CTEPH patients prior to right heart catheterization and PTE. Additionally, the increase in LA volume following PTE is significantly correlated with improvement in PVR, providing another potential non-invasive marker for postoperative success.

Our findings also provide evidence regarding the origin of LV diastolic dysfunction in CTEPH. This has been an area of research interest since PTE was developed as the definitive therapy for CTEPH [14,15]. In 1989, Dittrich et al. demonstrated improvement in LV diastolic filling with relief of chronic RV pressure overload [16]. In 2002, Mahmud et al. used the E/A ratio to show that early diastolic filling is primarily impaired in CTEPH, and that early LV filling improves with successful PTE [5]. In 2007, Gurudevan et al. evaluated whether the abnormal diastolic filling pattern $(\mathrm{E}<\mathrm{A})$ often seen in CTEPH was due to an intrinsic LV abnormality or impaired LV filling. The results suggested that that the abnormal E/A pattern was in large part due to decreased
LV preload [6]. These findings were supported by Lumens et al. using a computer-generated model that could separate the influences of septal bulging vs. LV filling on transmitral Doppler flow patterns pre- and post-PTE [7]. Gurudevan also reported that early diastolic mitral annular velocity (E') was abnormally low in CTEPH but increased after PTE. As in the present study, Gurudevan also showed that mitral annular E/E' rose significantly after PTE. Importantly, both E and E' increased postoperatively, with a greater proportion of increase in the $\mathrm{E}$ velocity [6].

As these studies are consistent with decreased preload as the cause of LV diastolic impairment in CTEPH, we decided to focus on the characteristics of the left atrium in this population. In this study, we found that smaller pre-operative LA volumes were significantly correlated with longer $\mathrm{E}$ wave deceleration times (i.e., impaired early diastolic filling). Furthermore, the subsequent increase in LA volume following PTE was significantly correlated with higher mitral E/A ratio. Both of these findings support the theory of LV underfilling and suggest that LA volume is a significant component of the process.

Whether LA volume in CTEPH is limited solely by decreased LA filling or by anatomic restrictions as well is not entirely clear. A recent study suggests that changes in RV outflow tract dimension may affect left atrial filling and compliance in CTEPH [17]. A proposed explanation is that the mediastinal space between the sternum and the spine is relatively fixed: an increase in RV and RV outflow tract size may limit normal expansion of the left atrium. Another recent report documented compression of the LA and RV by large hiatal hernias within the mediastinum [18]. This concept was demonstrated "in reverse" in a dog model, where pericardiectomy led to an increase in LA compliance, reservoir function, and early diastolic LV filling [19].

\section{Limitations}

A limitation in our study was the timing of the preoperative transthoracic echocardiogram and right heart catheterization. They were not performed simultaneously and in some instances up to 48 hours elapsed between procedures. As this population was stable preoperatively, it is unlikely this had a significant impact on the results. Post-operatively, echocardiography was performed an average of 9 days after PTE. It is conceivable that cardiac dimensions may have varied during this period, but again these patients were overall stable following surgery. As with several previous studies from our institution, echocardiography was delayed until patients left the surgical intensive care unit and could be examined safely in the noninvasive cardiac laboratory $[5,6,20]$. 


\section{Conclusions}

This study was designed to evaluate LA volume characteristics in CTEPH patients undergoing PTE. This data suggest that LA volume is inversely associated with severity of CTEPH, and that LA volume increases after PTE. The rapid increase in LAV and correlation with improvement in E/A ratio supports the concept that left ventricular diastolic impairment is due primarily to left heart underfilling rather than inherent left ventricular diastolic dysfunction. The long-term prognostic implications of LA volume change after PTE deserves further study.

\section{Abbreviations \\ CTEPH: Chronic thromboembolic pulmonary hypertension; LV: Left ventricle; LA: Left atrium; PTE: Pulmonary thromboendarterectomy; NYHA: New York Heart Association; UCSD: University of California San Diego; LAVI: Left atrial volume index; Cl: Cardiac index; RHC: Right heart catheterization; MPAP: Mean pulmonary artery pressure; PVR: Peripheral vascular resistance; PCWP: Pulmonary capillary wedge pressure; CO: Cardiac output.}

\section{Competing interests}

The authors declare that they have no competing interest.

\section{Authors' contributions}

All authors contributed to the study design, data collection, data analysis, and writing of the manuscript. All authors read and approved the final manuscript.

\section{Authors' information}

This manuscript originates from the Cardiovascular Center at the University of California, San Diego Medical Center, which has the highest-volume program for chronic thromboembolic pulmonary hypertension (CTEPH) and pulmonary thromboendarterectomy in the Western hemisphere. Dr. Blanchard has been senior author on a number of papers describing the interactions of the right and left ventricles in CTEPH over the last several years. Dr. Auger is a senior pulmonary specialist within the group, and Dr. Madani performs the majority of pulmonary thromboendarterectomies. Dr. Anthony DeMaria is the immediate past Editor-in-Chief of the Journal of the American College of Cardiology, and is a pre-eminent authority in echocardiography.

\section{Author details}

${ }^{1}$ School of Medicine and Sulpizio Cardiovascular Center, University of California San Diego, 9444 Medical Center Drive, \#7411, La Jolla, CA 92037, USA. ${ }^{2}$ Scripps Health, 501 Washington St. Ste 512, San Diego, CA 92103, USA.

Received: 23 May 2014 Accepted: 6 August 2014

Published: 11 August 2014

\section{References}

1. Fedullo P, Kerr KM, Kim NH, Auger WR: Chronic thromboembolic pulmonary hypertension. Am J Respir Crit Care Med 2011, 183:1605-1613.

2. Lang I: Advances in understanding the pathogenesis of chronic thromboembolic pulmonary hypertension. Br J Haemato/ 2012, 149:478-483.

3. Fedullo PF, Auger WR, Kerr KM, Rubin LJ: Chronic thromboembolic pulmonary hypertension. N Engl J Med 2001, 345(20):1465-1472.

4. Louie EK, Rich S, Brundage BH: Doppler echocardiographic assessment of impaired left ventricular filling in patients with right ventricular pressure overload due to primary pulmonary hypertension. J Am Coll Cardiol 1986, 8:1298-1306.

5. Mahmud E, Raisinghani A, Hassankhani A, Sadeghi M, Strachan G, Auger W, DeMaria AN, Blanchard DG: Correlation of left ventricular diastolic filling characteristics with right ventricular overload and pulmonary artery pressure in chronic thromboembolic pulmonary hypertension. J Am Coll Cardiol 2002, 40:318-324.

6. Gurudevan SV, Malouf PJ, Auger WR, Waltman TJ, Madani M, Raisinghani AB, DeMaria AN, Blanchard DG: Abnormal left ventricular diastolic filling in chronic thromboembolic pulmonary hypertension: true diastolic dysfunction or left ventricular underfilling? J Am Coll Cardiol 2007, 49:1334-1339.

7. Lumens J, Blanchard DG, Arts T, Mahmud E, Delhaas T: Left ventricular underfilling and not septal bulging dominates abnormal left ventricular filling hemodynamics in chronic thromboembolic pulmonary hypertension. Am J Physiol Heart Circ Physiol 2010, 299:1083-1091.

8. Thistlethwaite PA, Kaneko K, Madani MM, Jamieson SW: Technique and outcomes of pulmonary endarterectomy surgery. Ann Thorac Cardiovasc Surg 2008, 14:274-282.

9. Schiller NB, Shah PM, Crawford M, DeMaria A, Devereux R, Feigenbaum H, Gutgesell H, Reicheck N, Sahn D, Schnittger I: Recommendations for quantitation of the left ventricle by two-dimensional echocardiography. American Society of Echocardiography Committee on standards, subcommittee on quantitation of two-dimensional echocardiograms. J Am Soc Echocardiogr 1989, 2:358-367.

10. Tsang TS, Abhayaratna WP, Barnes ME, Miyasaka Y, Gersh BJ, Bailey KR, Cha SS, Seward JB: Prediction of cardiovascular outcomes with left atrial size: is volume superior to area or diameter? J Am Coll Cardiol 2006, 47:1018-1023.

11. Wessa P: Free Statistics Software, Office for Research Development and Education, version 1.1.23-r7; 2013. URL http://www.wessa.net/.

12. Hoit BD: Left atrial size and function: role in prognosis. $J$ Am Coll Cardiol 2014, 63:493-505.

13. Barbier P, Solomon SB, Schiller NB, Glantz SA: Left atrial relaxation and left ventricular systolic function determine left atrial reservoir function. Circulation 1999, 100:427-436.

14. Thistlethwaite PA, Madani MM, Jamieson SW: Outcomes of pulmonary endarterectomy surgery. Semin Thorac Cardiovasc Surg 2006, 18:257-264.

15. Moser KM, Daily PO, Peterson KL, Dembitsky W, Vapnek JM, Shure D, Utely J, Archibald C: Thromboendarterectomy for chronic, major-vesse thromboembolic pulmonary hypertension: immediate and long-term results in 42 patients. Ann Intern Med 1987, 107:560-565.

16. Dittrich HC, Chow LC, Nicod PH: Early improvement in left ventricular diastolic function after relief of chronic right ventricular pressure overload. Circulation 1989, 80:823-830.

17. Kimura BJ, Parise C, Strachan GM, Auger WR, Madani MM, Daniels LB, Blanchard DG: Diminished aortic excursion in chronic thromboembolic pulmonary hypertension. Echocardiography 2013, 30:1126-1129.

18. Naoum C, Falk GL, Ng ACC, Lu T, Ridley L, Ing AJ, Kritharides L, Yiannikas J: Left atrial compression and the mechanism of exercise impairment in patients with large hiatal hernia. J Am Coll Cardiol 2011, 58:1624-1634.

19. Hoit BD, Shao Y, Gabel M, Walsh RA: Influence of pericardium on left atrial compliance and pulmonary venous flow. Am J Physiol 1993, 264:H1781-H1787.

20. Blanchard DG, Malouf PJ, Gurudevan SV, Auger WR, Madani MM, Thistlethwaite P, Waltman TJ, Daniels LB, Raisinghani AB, DeMaria AN: Utility of right ventricular Tei index in the noninvasive evaluation of chronic thromboembolic pulmonary hypertension before and after pulmonary thromboendarterectomy. J Am Coll Cardiol Img 2009, 2:143-149.

doi:10.1186/1476-7120-12-32

Cite this article as: Marston et al:: Assessment of left atrial volume before and after pulmonary thromboendarterectomy in chronic thromboembolic pulmonary hypertension. Cardiovascular Ultrasound 2014 12:32 